\title{
ANÁLISIS DE LA MORTALIDAD PERINATAL, HOSPITAL MIGUEL SERVET, ZARAGOZA, ESPAÑA: 2000-2009
}

\author{
Esther Cruz G. 1,2, María Lapresta M. 1,2, Raquel Crespo E. 1,2, Yasmina José G. 1, \\ Pilar Andrés O. ${ }^{1,2}$, Javier Tobajas H. ${ }^{1,2}$ \\ ${ }^{1}$ Servicio de Obstetricia, Hospital Universitario Miguel Servet, ${ }^{2}$ Instituto Aragonés de Ciencias de la Salud, Zaragoza, \\ España.
}

\section{RESUMEN}

Objetivos: Evaluar la mortalidad perinatal (MP) del Hospital Miguel Servet (2000-2009). Valorar su evolución, distribución según edad gestacional y peso de los recién nacidos (RN), y las posibles causas. Método: Análisis sobre un total de $44.409 \mathrm{RN} \geq 500$ gramos y 402 nacidos muertos ó fallecidos antes de los 28 días completos de vida. Los datos se recogieron en un formulario siguiendo las recomendaciones de la FIGO y la novena Clasificación Internacional de Enfermedades. Resultados: La MP ampliada global fue de 9,05/1000 nacidos, la mortalidad fetal de 4,91/1000 nacidos y la mortalidad neonatal 4,14/1000 nacidos vivos. La MP estándar fue de 4,73/1000 nacidos. Según la edad gestacional, un $79,6 \%$ de la MP fueron pretérminos; el $46,02 \%$ de los casos eran $<1000$ gramos y $59,95 \%<1500$ gramos. Procedían de partos múltiples 92 RN (90 pretérminos); la MP de los embarazos gemelares fue 34,65/1000 nacidos. Las causas más frecuentes de MP eran: inmadurez extrema, $<1000$ gramos $(46,02 \%)$, rotura prematura de membranas $(23,13 \%)$, gestaciones múltiples $(22,89 \%)$, complicaciones de placenta o cordón $(21,64 \%)$, hipoxia intrauterina y asfixia al nacer $(21,64 \%)$, y malformaciones congénitas y cromosomopatías $(18,9 \%)$. Conclusión: La MP está gravada por la prematuridad, el bajo peso extremo y los embarazos múltiples, disminuyendo paulatinamente a expensas de la mortalidad neonatal.

PALABRAS CLAVE: Mortalidad perinatal, mortalidad fetal, mortalidad neonatal, estadísticas vitales

\section{SUMMARY}

Objective: To evaluate Hospital Miguel Servet perinatal mortality (PM) during the years 2000-2009, valuing its evolution and distribution according to gestational age and birth weight. Maternal and fetal possible causes are analized. Methods: 44.409 newborns of $\geq 500$ grams or 22 weeks gestational age, were studied. The total number of perinatal deaths was 402 containing late neonatal deaths (to 28 completed days of live). The data for each case of perinatal mortality were recorded in a form following FIGO recommendations and the 9th International Classification of Diseases. Results: Extended PM rate was 9.05/1000 newborns, fetal mortality 4.91/1000 newborns and neonatal mortality 4.14/1000 live births. Standard PM was 4.73/1000 newborns. According to gestational age, $79.60 \%$ were preterm deliveries. Considering birth weights, $46.02 \%$ were extremely low birth weight ( $<1000$ grams), and $59.95 \%$ were $<1500$ grams. The number of perinatal deaths in multiple pregnancies was 92 (90 preterm deliveries). PM in twins was 34.65/1000 newborns. Main causes of death were extreme immaturity $(<1000$ grams) in $46.02 \%$, premature rupture of membranes (23.13\%), multiple deliveries $(22.89 \%)$, complications of placenta or umbilical cord $(21.64 \%)$, intrauterine hypoxia and asphyxia at birth in $21.64 \%$ of the cases, and congenital malformations and chromosomopaties 
(18.90\%). Conclusions: PM is encumbered by prematurity, extremely low birth weight and multiple pregnancies. Decrease of perinatal mortality had occurred mainly at the expense of neonatal mortality.

\section{KEY WORDS: Perinatal mortality, fetal mortality, neonatal mortality, vital statistics}

\section{INTRODUCCIÓN}

La mortalidad perinatal es uno de los indicadores básicos que miden la calidad de la asistencia obstétrica. Continúa siendo el mejor indicador de salud durante el periodo perinatal, ya que generalmente está disponible. Es un índice excesivamente grosero que ignora los numerosos factores que pueden estar asociados con las muertes perinatales, como la tasa de mortalidad por grupos de pesos, de edad gestacional al nacer, la causa de la muerte, o la importancia relativa de cada causa de muerte en la población. No identifica las muertes perinatales que son potencialmente evitables, ni discrimina aquellas causadas por anomalías congénitas incompatibles con la vida (1). Por otra parte, todavía sigue habiendo grandes dificultades en la comparación de los datos entre los distintos países e incluso entre distintos centros en cada país por variaciones en la forma de recogerlos y comunicarlos $(2,3,4)$.

El objetivo del presente trabajo es evaluar la mortalidad perinatal de los últimos 10 años $(2000$ - 2009) habida en el Servicio de Obstetricia del Hospital Universitario Miguel Servet de Zaragoza, España, analizando su evolución, la distribución según edad gestacional y pesos, y las posibles causas tanto maternas como fetales que pudieran estar implicadas.

\section{MATERIAL Y MÉTODO}

Estudio descriptivo, de tipo retrospectivo, sobre 43.224 partos ocurridos entre el 1 de enero de 2000 y el 31 de diciembre de 2009, en el Hospital Universitario Miguel Servet (HUMS) de Zaragoza. Sobre un total de 44.409 recién nacidos (RN) de más de 22 semanas completas de vida o de 500 o más gramos de peso, se evaluaron aquellos que nacieron muertos ó fallecieron antes de los 28 días completos de vida (mortalidad perinatal ampliada). Los datos de cada caso de mortalidad perinatal (MP) se recogieron en una ficha - formulario confeccionada específicamente para este estudio, que analizaba criterios sociodemográficos, paridad previa, origen, datos sobre evolución de la gestación actual, tipo de parto y posibles complicaciones, y todos los valores perinatales que se recogían de forma protocolizada en todos los nacimientos. Se transcribieron asimismo las posibles causas implicadas, tanto maternas como fetales, la existencia de malformaciones congénitas, si se realizó necropsia, y en caso de mortalidad neonatal, las complicaciones de este periodo.

La codificación del formulario se realizó siguiendo las recomendaciones de la FIGO y de la novena Clasificación Internacional de Enfermedades (CIE9). Los datos se introdujeron en una base informatizada diseñada para esta investigación, mediante el programa Microsoft Access 2003 y el estudio descriptivo se realizó con el programa Microsoft Excel 2003 (Microsoft $\circledast$ Office 2003; Copyright $@$ 19832003 Microsoft Corporation).

Se revisaron las bases de datos de ámbito general Pubmed y Embase, insistiendo en las publicaciones europeas, así como revistas médicas en lengua castellana, especialmente españolas, de Obstetricia y Ginecología, desde 2003 hasta 2009.

\section{RESULTADOS}

De los $44.409 \mathrm{RN}$ durante los diez años analizados en este estudio, hubo 402 que nacieron muertos o fallecieron antes de los 28 días completos de vida, lo que supuso una MP ampliada global de 9,05/1000 nacidos. Para comparaciones internacionales, que habitualmente tienen en cuenta las gestaciones de 28 o más semanas y hasta 7 días completos de vida (MP estándar o MP tipo I) la MP I fue de 4,73/1000 nacidos.

La MP anual fue descendiendo paulatinamente durante el periodo de estudio, salvo los dos últimos años, en que el descenso ha sido más notorio (Figura 1). La mortalidad fetal ampliada fue de $4,91 / 1000$ nacidos y la mortalidad neonatal ampliada de 4,14/1000 nacidos vivos. La mortalidad fetal y la mortalidad neonatal anual se muestran en la Tabla I.

La distribución de la MP según la edad gestacional se detalla en la Tabla II, objetivándose que el $79,6 \%$ correspondió a pretérminos. Un $53,23 \%$ de la MP fue a expensas de nacidos con edad gestacional menor de 32 semanas, sin embargo, estas gestaciones solo constituyeron aproximadamente el $2 \%$ del total de partos atendidos en nuestro hospital. 
Tabla I

EVOLUCIÓN DE LA MORTALIDAD FETAL Y NEONATAL EN EL HOSPITAL UNIVERSITARIO MIGUEL SERVET, 2000-2009 (ZARAGOZA, ESPAÑA)

\begin{tabular}{|c|c|c|c|c|c|c|c|c|c|c|}
\hline \multirow[t]{2}{*}{ Mortalidad } & \multicolumn{10}{|c|}{ AÑOS } \\
\hline & 2000 & 2001 & 2002 & 2003 & 2004 & 2005 & 2006 & 2007 & 2008 & 2009 \\
\hline Fetal * & 5,41 & 5,44 & 5,79 & 3,51 & 4,73 & 5,37 & 6,11 & 4,78 & 4,34 & 5,02 \\
\hline Neonatal ** & 6,45 & 4,56 & 4,13 & 5,14 & 3,83 & 4,06 & 5,24 & 3,78 & 1,38 & 1,68 \\
\hline
\end{tabular}

${ }^{*} \times 1000$ nacidos. ${ }^{* *} \times 1000$ nacidos vivos.

MORTALIDAD PERINATAL 2000-2009

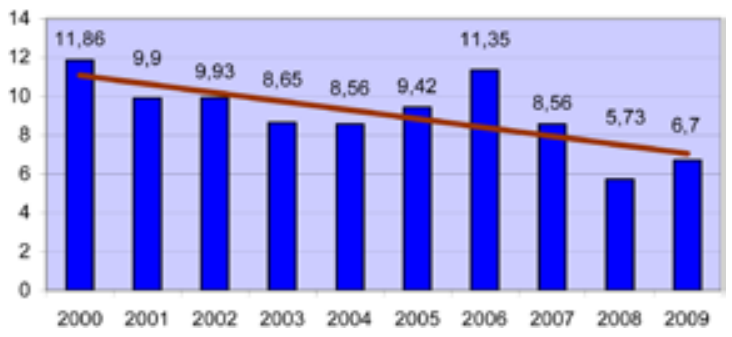

Figura 1. Evolución de la tasa de mortalidad perinatal ampliada en el Hospital Universitario Miguel Servet, 2000-2009 (Zaragoza, España).

Tabla II

DISTRIBUCIÓN DE LA MORTALIDAD PERINATAL SEGÚN LA EDAD GESTACIONAL AL NACER

\begin{tabular}{ccc}
\hline $\begin{array}{c}\text { Edad gestacional } \\
\text { (semanas) }\end{array}$ & Casos & $\%$ \\
\hline $22-27$ & 154 & 38,31 \\
$28-31$ & 87 & 21,64 \\
$32-36$ & 79 & 19,65 \\
$\geq 37$ & 82 & 20,40 \\
\hline
\end{tabular}

La distribución según peso al nacer muestra que la MP está gravada por la gran inmadurez de los fetos: $46,02 \%$ correspondieron a $<1000$ gramos y $59,95 \%$ a $R N<1500$ gramos (Tabla III).

En cuanto al tipo de parto (Figura 2), el $46 \%$ fueron eutócicos, siendo la tasa de cesáreas de $35 \%$. Durante el periodo de estudio, la tasa global de cesáreas del HUMS de Zaragoza fue de 16,5\%.

Un 22,89\% (92/402) de los casos de MP procedían de partos múltiples, cuya distribución por número de nacidos y semanas de gestación se muestra en la Tabla IV. Todos los casos de muer- te perinatal en embarazo múltiple presentaron una edad gestacional menor de 37 semanas, salvo dos casos: una gestación gemelar con muerte súbita del $2^{\circ}$ gemelo en el posparto y un caso de embarazo múltiple con un feto afecto por una trisomía 18 que falleció in útero.

Tabla III

\section{DISTRIBUCIÓN DE LA MORTALIDAD PERINATAL SEGÚN PESO AL NACER}

\begin{tabular}{ccc}
\hline $\begin{array}{c}\text { Peso } \\
\text { (gramos) }\end{array}$ & Casos & $\%$ \\
\hline $500-999$ & 185 & 46,02 \\
$1000-1499$ & 56 & 13,93 \\
$1500-1999$ & 39 & 9,70 \\
$2000-2499$ & 35 & 8,71 \\
$\geq 2500$ & 87 & 21,64 \\
\hline
\end{tabular}

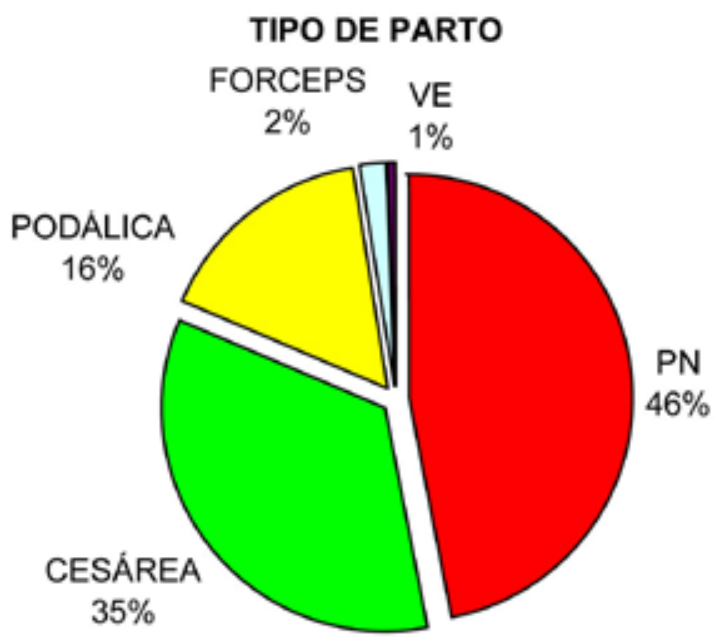

Figura 2. Distribución de la mortalidad perinatal según el tipo de parto. 
Tabla IV

EMBARAZO MÚLTIPLE Y MORTALIDAD PERINATAL SEGÚN EDAD GESTACIONAL AL NACER

\begin{tabular}{ccccc}
\hline Semanas & Gemelares & Triples & Cuádruples & Total $(\%)$ \\
\hline$<28$ & 39 & 10 & 4 & $53(58,61)$ \\
$28-31$ & 22 & 2 & 1 & $25(27,17)$ \\
$32-36$ & 9 & 3 & - & $12(13,04)$ \\
$37-41$ & 2 & - & - & $2(2,17)$ \\
$\geq 41$ & - & - & - & - \\
\hline
\end{tabular}

Entre los años 2000-2009 se atendieron 1.101 partos múltiples, lo que supuso un total de 2.267 recién nacidos de embarazos multifetales. La MP de los embarazos múltiples fue de 40,58/1000 nacidos $(34,65 / 1000$ nacidos en gestaciones gemelares y $84,74 / 1000$ nacidos en triples).

Hubo 76 casos de muerte perinatal con malformaciones congénitas, 7 de ellas correspondieron a cromosomopatías (Tabla V). En 42 casos las malformaciones eran incompatibles con la vida $(55,26 \%)$ y en varias ocasiones coexistieron malformaciones múltiples en un mismo neonato.

Una parte importante de las gestaciones que derivaron en MP provenían de otras áreas hospitalarias (Figura 3). Fueron 85 los casos procedentes de derivaciones, por necesidades de cuidados intensivos neonatales y/o cirugía neonatal compleja, y supusieron el $21,14 \%$ de la MP en nuestro centro. Así, la MP ampliada ajustada a nuestra área de influencia sería de 6,46/1000 nacidos.

Dado el importante crecimiento en los últimos años de la población inmigrante atendida en nuestro centro (aproximadamente el $20 \%$ de los partos), desde el año 2006 recogemos datos sobre la procedencia de cada paciente en el formulario de mortalidad perinatal. Incluido el año 2006, hubo 19.202 recién nacidos, con 50 casos de MP de madres extranjeras, lo que supuso un $32,42 \%$ de la MP global.

Al analizar las causas básicas de muerte perinatal (CIE-9), se intentó separar las mismas en causas maternas y causas fetales. Las causas más frecuentes, determinadas por necropsia (215 necropsias; $53,48 \%$ de los casos) ó por criterios clínicos se muestran en la Tabla VI. Entre las complicaciones del embarazo destaca con 93 casos ( $23,18 \%$ del total) la rotura prematura de membranas, que en 29 casos evolucionó a corioamnionitis y muerte perinatal. Entre las causas neonatales, la más frecuente fue la inmadurez extrema $(<1000$ gramos $0<28$ semanas), con 185 casos $(46,02 \%)$.
Tabla V

\section{ESTUDIO DE LAS MALFORMACIONES CONGÉNITAS ENCONTRADAS EN LOS FETOS OBJETO DE ESTUDIO SEGÚN CIE-9} (76 CASOS)

\begin{tabular}{llc}
\hline CIE-9 & Malformaciones congénitas & Casos \\
\hline 740 & Anencefalia y anomalías similares & 2 \\
742 & $\begin{array}{l}\text { Otras anomalías congénitas sistema } \\
\text { nervioso central }\end{array}$ & 11 \\
745 & Anomalías bulbo arterioso y cierre septal & 14 \\
746 & Otras anomalías congénitas del corazón & 10 \\
747 & $\begin{array}{l}\text { Otras anomalías congénitas del aparato } \\
\text { circulatorio* }\end{array}$ & 8 \\
748 & $\begin{array}{l}\text { Anomalías congénitas aparato respira- } \\
\text { torio }\end{array}$ & 3 \\
750 & $\begin{array}{l}\text { Otras anomalías congénitas aparato } \\
\text { digestivo superior }\end{array}$ & 7 \\
753 & $\begin{array}{l}\text { Anomalías congénitas del aparato } \\
\text { urinario }\end{array}$ & 8 \\
754 & Anomalías osteomusculares congénitas \\
755 & $\begin{array}{l}\text { Otras anomalías congénitas de los } \\
\text { miembros }\end{array}$ & 4 \\
756 & $\begin{array}{l}\text { Otras anomalías congénitas del sistema } \\
\text { osteomuscular }\end{array}$ & 3 \\
759 & Otras no especificadas y múltiples & 4 \\
758 & Anomalías cromosómicas & 7 \\
\hline
\end{tabular}

* No se incluyen la arteria umbilical única ni la persistencia del conducto arterioso.

\section{PROCEDENCIA}

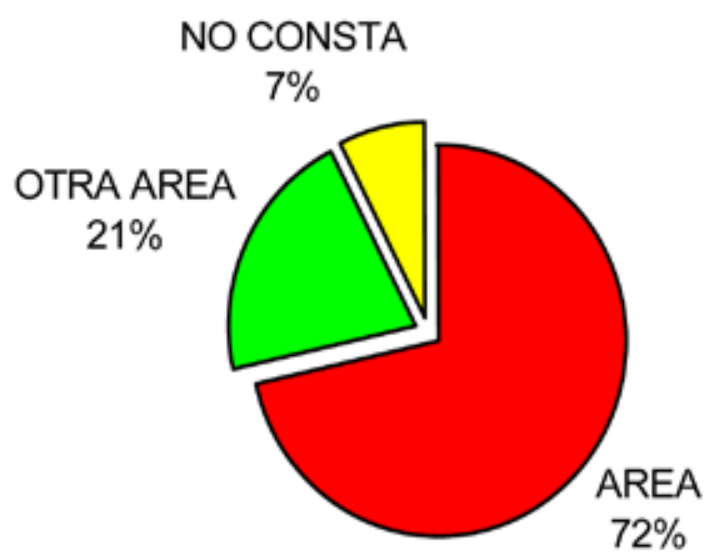

Figura 3. Distribución de los casos de mortalidad perinatal según pertenezcan al área de influencia del HUMS de Zaragoza. 
Tabla VI

\section{CAUSAS MÁS FRECUENTES ASOCIADAS A MORTALIDAD PERINATAL (MATERNAS, FETALES, NEONATALES Y OVULARES)}

\begin{tabular}{lc}
\hline Causas & Casos * $(\%)^{\circ}$ \\
\hline $\begin{array}{l}\text { Inmadurez extrema }<1000 \mathrm{~g} /<28 \\
\text { semanas }\end{array}$ & $185(46,02)$ \\
RPM (29 corioamninitis) & $93(23,13)$ \\
Embarazo múltiple & $92(22,89)$ \\
$\begin{array}{l}\text { Feto o RN afecto por complicaciones } \\
\text { de la placenta/cordón }\end{array}$ & $87(21,64)$ \\
$\begin{array}{l}\text { Hipoxia intrauterina } \\
\text { Malformaciones congénitas y } \\
\text { cromosomopatías }\end{array}$ & $87(21,64)$ \\
\end{tabular}

* Algunos casos presentan 2 o más causas. RN: recién nacido. RPM: rotura prematura de membranas.

\section{DISCUSIÓN}

La MP ha disminuido muy paulatinamente durante el periodo evaluado (excepto en los dos últimos años) en contraste con la evidente disminución hallada en años anteriores en nuestro centro. Esta disminución es una tendencia que se observa en muchas publicaciones (5-7) pero es muy gradual, e interpretamos que es debida al incremento de partos pretérmino que lentamente se está produciendo, hecho al que no son ajenas las técnicas de reproducción asistida, y que en esta década se han afianzado en nuestro Hospital.

Cada vez son mayores los nacimientos de RN de muy bajo peso y de bajo peso extremo con su consiguiente riesgo de morbimortalidad, a lo que contribuye también el mayor número de gestaciones múltiples (8-11). Vemos de forma clara que la disminución de la MP es a expensas de la mortalidad neonatal; la mortalidad fetal se mantiene prácticamente constante, como comunican otros autores (12).

La menor mortalidad neonatal interpretamos puede ser debida a la mejora continua en los cuidados neonatales, incluyendo el perfeccionamiento de la tecnología empleada en los cuidados intensivos pediátricos (empieza a haber en las estadísticas pediátricas datos de mortalidad posneonatal, referida a la mortalidad ocurrida después de los 28 días de vida), al cribado prenatal universal de aneuploidías y malformaciones, e incluso, a la mejora continua en los controles obstétricos prenatales, que discriminan cada vez mejor los embarazos de riesgo. La mortalidad fetal tiene que seguir siendo motivo de estudio para lograr incidir en ella, e intentar lograr minimizar la incidencia de causas de muerte "inexplicables" $(12,13)$.
En nuestro país la uniformidad es ya habitual en la terminología, y a ello ha contribuido en gran medida la Encuesta Nacional de Mortalidad Perinatal realizada por la Sección de Medicina Perinatal de la Sociedad Española de Ginecología y Obstetricia (SEGO) que se realiza desde 1980 (14) y, en general, nos expresamos siempre en términos de MP ampliada ó MPII, aunque a veces, los mismos datos, según sea la fuente que los divulgue, pueden no coincidir $(4,5)$.

En las publicaciones internacionales se utiliza más comúnmente la mortalidad perinatal estándar, que incluye los fetos de $\geq 28$ semanas ó $\geq 1000$ gramos, no incluyéndose la que corresponde a gestaciones entre 22 y 28 semanas ni la mortalidad neonatal tardía (más de 7 y hasta 28 días de vida) (15). Incluso hemos encontrado valoraciones y tasas intermedias tales como mortalidad fetal ampliada (desde las 22 semanas completas de gestación) con mortalidad neonatal precoz (hasta 7 días completos de vida) $(16,17)$. Todo ello contribuye a que las comparaciones internacionales resulten difíciles.

En Europa se inició en 1996 el Euronatal Study, que incluía 14 países, y que se ha ido ampliando y modificando, concretándose en el "Peristat Project" $(18,19)$, y que selecciona un conjunto mínimo de indicadores para monitorizar y evaluar la salud perinatal en Europa $(20,21)$. Participan en el momento actual un total de 26 países, y se han comunicando resultados en los informes Peristat I y Peristat II $(16,22)$. Es un serio intento de estudio de las condiciones perinatales de cada país y valida la MP nacional como indicador de la calidad de la atención antenatal y perinatal. Además podría ayudar a unificar la agrupación de datos, que aún hoy se dan de forma discordante.

El Servicio de Neonatología del HUMS es centro de referencia (nivel terciario), además de para nuestra área de influencia, para gran parte de la Comunidad Autónoma de Aragón, para la Comunidad Autónoma de La Rioja, para la Provincia de Soria, y para todos aquellos RN de Aragón que precisen cirugía compleja con cuidados intensivos. Por ello, la mortalidad perinatal de nuestro hospital se ha visto incrementada con la que aportan los nacimientos en el Servicio de Obstetricia de estos casos trasladados de otras áreas, algunas con su hospital de referencia de nivel terciario. Al menos un $21,14 \%$ de nuestra MP está gravada por traslados de otras áreas.

Siguen surgiendo nuevos trabajos valorando las características de los centros de nivel terciario y su influencia en la MP exponiendo resultados (23), algunos tan llamativos, como que solo es significativo en cuanto a MP la asistencia de nivel terciario por debajo de las 30 semanas de gestación (24). 
Las tasas de MP son diferentes no sólo entre distintos países $(15,16,25,26)$, sino incluso dentro de un mismo país, hecho relacionado con la calidad y el nivel de los cuidados ofertados. Se han descrito tasas diferentes según la distribución racial de la población atendida en centros de un mismo país, y en este punto hay múltiples publicaciones referidas a la inmigración (8,27-30). También se han comunicados diferencias en una misma raza, según el área geográfica considerada (31). A pesar de los avances en nuestra especialidad, se sigue manteniendo la tasa de MP como un indicador clave de la calidad asistencial, y todas estas diferencias nos hacen pensar que además de otros factores fundamentales, como los sociales y ambientales, los biológicos, o los factores fetales y del recién nacido, se nace con distintas posibilidades según la atención recibida durante la gestación y la dotación del centro al que se tenga acceso (32).

Están apareciendo datos recientes en las publicaciones internacionales sobre la MP de los partos múltiples (33), en comparación con el casi silencio de épocas anteriores, y es que el número de ellos se ha visto muy incrementado por el desarrollo de las técnicas de reproducción asistida. Cuando se comparan con las gestaciones simples, diferentes autores han comunicado el hallazgo de una tasa superior de MP en fetos de embarazos múltiples menores de 2000 gramos, pretérminos ó con edad materna extrema (menor de 20 y mayor de 40 años).

En cuanto a las causas básicas de MP, hay unanimidad en que la inmadurez extrema es de las más frecuentes, así como la hipoxia intrauterina y la asfixia al nacer. Se refieren múltiples causas maternas y complicaciones del embarazo, destacando la rotura prematura de membranas (en nuestro caso un $23,13 \%)$, las gestaciones múltiples y la patología placentaria. Es importante resaltar que en la población analizada en este estudio las alteraciones funiculares y placentarias supusieron un $21,64 \%$ del total.

Hemos encontrado que, a pesar de todo, sigue existiendo un número importante de muertes fetales inexplicables, lo que contribuye a que la mortalidad fetal permanezca casi inalterable a lo largo de los últimos años. Este hallazgo coincide con el reflejado por otros autores $(12,34)$. Posiblemente, en los próximos años, uno de los retos de la investigación en obstetricia sea analizar las causas de mortalidad fetal y definir métodos de mejora en la asistencia, que permitan disminuir las tasas globales de mortalidad perinatal.

\section{CONCLUSIÓN}

La MP está gravada por la prematuridad $(79,6 \%)$, el bajo peso extremo $(46,02 \%)$ y los em- barazos múltiples $(22,89 \%)$, disminuyendo paulatinamente a expensas de la mortalidad neonatal.

\section{BIBLIOGRAFÍA}

1. Fabre González E, González de Agüero Laborda R, Pérez Hiraldo P. Concepto y división de la patología perinatal. En: Cabero Roura L, Tratado de Ginecología, Obstetricia y Medicina de la Reproducción. Editorial Médica Panamericana. Madrid 2003, 848-854.

2. Cruz E, Lapresta M, José Y, Andrés P, Villacampa A. Mortalidad perinatal. Distribución según la edad gestacional y el peso al nacimiento. Causas más frecuentes. Revisión epidemiológica de 10 años. Clin Invest Gin Obst 2004; 31(9):323-7

3. Lack N, Zeitlin J, Krebs L, Künzel W, Alexander S. Methodological difficulties in the comparison of indicators of perinatal health across Europe. Eur J Obstet Gynecol Reprod Biol 2003;111 Suppl 1:S33-44.

4. Freitas A, Puig A Gispert R, Marcano M, Bustins M. La mortalidad perinatal según 2 fuentes de información. Gac Sanit 2008;22:378-81.

5. Aceituno L, Huertas M, Sánchez MT, Ruiz E, Delgado L, González V, et al. Resultado de la atención perinatal en el 2007. Evolución en el último decenio. Prog Obstet Ginecol 2010;53:133-40.

6. Díaz V, Berzosa J, Alonso E. Mortalidad, morbilidad inmediata y factores de riesgo en recién nacidos con extremado bajo peso al nacimiento. Prog Obstet Ginecol 2005;48:165-711.

7. De la Puente M, Izquierdo N, Coronado PJ, Alonso T, Armada MI. Mortalidad perinatal en el Hospital Clínico San Carlos de Madrid. Prog Obstet Ginecol 2009;52:386-92.

8. MacDorman MF, Munson ML, Kirmeyer S. Fetal and perinatal mortality, United States, 2004. Nat Vital Stat Rep 2007;56:1-20.

9. Evans N, Hutchinson J, Simpson JM, Donoghue D, Darlow B, Henderson-Smart D. Prenatal predictors of mortality in very preterm infants cared for in the Australian and New Zealand Neonatal Network. Archives of Disease in Childhood: Fetal and Neonatal Edition 2007;92:F34-F40.

10. Newman DE, Paamoni-Keren O, Press F, Wiznitzer A, Mazor M, Sheiner E. National outcome in preterm deliveries between 23 and 27 weeks' gestation with and without preterm premature rupture of membranes. Arch Gynecol Obstet 2009;280:7-11.

11. Papiernik E, Zeitlin J, Delmas D, Draper ES, Gadzinowski J, Künzel W, et al. Termination of pregnancy among very preterm births and its impact on very preterm mortality: Results from ten European population-based cohorts in the MOSAIC study. BJOG 2008;115:361-8.

12. Walsh CA, Vallerie AM, Baxi LV. Etiology of stillbirth at term: A 10-year cohort study. J Matern Fetal Neonatal Med 2008;21:493-501.

13. Lu JR, McCowan IA comparison of Perinatal Society of Australia and New Zealand-Perinatal Death Classification system and relevant condition at death stillbirth classification systems. Aust N Z Obstet Gynaecol 2009;49:467-71. 
14. González NL, Medina V, Jiménez A, Gómez J, Ruano A, Perales A, et al. Base de datos perinatales nacionales del año 2004. Prog Obstet Ginecol 2006;49:645-55.

15. Buitendijk SE, Nijhuis JG. High perinatal mortality in the Netherlands compared to the rest of Europe. Ned Tijdschr Geneeskd 2004;148:1853-5.

16. Mohangoo AD, Buitendijk SE, Hukkelhoven CW, Ravelli $A C$, Rijninks-van Driel GC, Tamminga $P$, Nijhuis JG. Higher perinatal mortality in The Netherlands than in other European countries: the Peristat-II study. Ned Tijdschr Geneeskd 2008;152:2718-27.

17. Ravelli AC, Eskes M,Tromp M, Van Huis AM,Steegers EA,Tamminga $P$ et al. Perinatal mortality in The Netherlands 2000-2006; risk factors and risk selection. Ned Tijdschr Geneeskd. 2008;152:2728-33.

18. Editorial: Perinatal health indicators for Europe: an introduction to the PERISTAT project. Eur J Obstet Gynecol Reprod Biol 2003;111:S1-S4.

19. Zeitlin J, Wildman K, Bréart G, Alexander S, Barros H, Blondel $\mathrm{B}$, et al. PERISTAT: Indicators for monitoring and evaluating perinatal health in Europe. Eur J Public Health 2003;13(3 Suppl):29-37.

20. Zeitlin J, Wildman K, Bréart G, Alexander S, Barros H, Blondel $\mathrm{B}$, et al. Selecting an indicator set for monitoring and evaluating perinatal health in Europe: Criteria, methods and results from the PERISTAT project. Eur J Obstet Gynecol Reprod Biol 2003;111:S5-S14.

21. Buitendijk S, Zeitlin J, Cuttini M, Langhoff-Roos J, Bottu J. Indicators of fetal and infant health outcomes. Eur J Obstet Gynecol Reprod Biol 2003;111:S66-S67.

22. European Perinatal Health Report by the Euro Peristat project.Date from 2004. Perinatal Health report 2008 [en línea]. Disponible en: www.europeristat.com [Consulta: 12 Agosto 2010].

23. Bartels DB, Wenzlaff $P$, Poets CF. Obstetrical volume and early neonatal mortality in preterm infants. Eur $\mathrm{J}$ Epidemiol 2007;22:791-8.

24. Waner B, Musial MJ, Chenier T, Donovan E. The effect of birth hospital type on the outcome of very low birth weigth infants. Pediatrics 2004;113:35-41.

25. Carlo WA, Goudar SS, Jehan I, Chomba E,Tshefu A, Garces A, et al. Newborn-care training and perinatal mortality in developing countries. $\mathrm{N}$ Engl J Med 2010;362:614-23.

26. Borrell C, Cirera E, Ricart M, Pasarín MI, Salvador J. Social inequalities in perinatal mortality in a Southern European city. Eur J Epidemiol 2003;18:5-13.

27. García J, Pardo C, Hernández A, Lorenzo M. Diferencias obstétricas y neonatales entre mujeres autóctonas e inmigrantes. Prog Obstet Ginecol 2008;51:53-62.

28. Gagnon AJ, Zimbeck M, Zeitlin J. Migration and perinatal health surveillance: An international Delphi survey. Eur J Obstet Gynecol Reprod Biol 2010;149:37-43.

29. Alderliesten ME, Stronks K, Van Lith JMM, Smit BJ, Van der Wal MF, Bonsel GJ, Bleker OP. Ethnic differences in perinatal mortality. A perinatal audit on the role of substandard care. Eur J Obstet Gynecol Reprod Biol 2008;138:164-70.

30. Racape J, De Spiegelaere M, Alexander S, Dramaix M, Buekens P, Haelterman E. High perinatal mortality rate among immigrants in Brussels. Eur J Public Health 2010;20:536-42.

31. Tromp M, Eskes M, Reitsma JB, Erwich JJ, Brouwers $\mathrm{H}$, Rijninks-van Driel G, et al. Regional perinatal mortality differences in the Netherlands; care is the question. BMC Public Health 2009,9:102 (doi:10.1186/14712458-9-102).

32. Martín-Martínez A, García-Hernández JA. Índice de riesgo gestacional, vía de parto y resultados perinatales. Prog Obstet Ginecol 2005;48:231-8.

33. Hernández RJ, del Rayo Y, Alcalá LG, Ran R, Flores R, Torcida ME. Mortalidad perinatal en embarazos múltiples. Ginecol Obstet Mex 2009;77:147-50.

34. Valdés E, Toledo V, Preisler J. Muerte fetal: experiencia de 10 años en el Hospital Clínico de la Universidad de Chile. Prog Obstet Ginecol 2008;51:656-64. 